\section{A magnitude analysis of differential classical, avoidance, and classical-avoidance eyelid conditioning in the monkey (Cebus Albifrons) ${ }^{1}$}

\author{
$M I C H A E L$ A. MILAN and H. S. \\ PENNYPACKER, University of Florida, \\ Gainesville, Fla. 32601
}

Cebus monkeys were subjected to differential classical, differential avoidance, or differential classical-avoidance eyelid conditioning. Conditioning performance was analyzed in terms of both response probability and eyeblink magnitude. The response-probability analysis failed to reveal either acquisition or discrimination under any treatment condition. The magnitude analysis demonstrated differentiation in the within-Ss comparison of the two conditioning paradigms and suggested differentiation in the differential-avoidance group.

In a recent experiment, Massaro \& Moore (1967) compared differential classical, differential avoidance, and differential classical-avoidance conditioning of the human eyelid response. Examination of response-probability data revealed significant differentiation under both the differential-classical and differential-avoidance paradigms. In addition, the topographies of the responses appeared to differ, with the classically conditioned responses demonstrating faster recruitment and longer duration than the avoidance responses. However, responses generated by the two contingencies in the within-Ss comparison of the two paradigms failed to differ in either response probability or response topography, leading Massaro and Moore to the conclusion that the two responses cannot develop simultaneously within the same $S$.

The present study is both a replication of the Massaro and Moore study, utilizing infrahuman primates as $\mathrm{Ss}$, and an extension, for a response-magnitude measure is introduced to supplement the commonly employed dependent variable, response probability. A number of considerations underlie the employment of the new measure: First, although the independent variables influencing conditioning of the eyelid response have been extensively examined, with few exceptions (e.g., Fishbein \& Gormezano, 1966; Levey \& Martin, 1965), researchers in this area have concentrated their attention upon only one of several available dependent variables, response frequency, or its commonly employed derivative, response probability. The relationships between frequency measures and other response measures, such as response amplitude, area, latency, and magnitude, are largely undetermined. In what fashion these potential indicators of the conditioning process vary as a function of experimental variables is virtually unknown. It is not expected that these various measures will reflect in a homomorphic fashion the effects of manipulation of different independent variables, for it is axiomatic that different response measures are differentially sensitive to different processes. However, until such experimentation is completed, general statements concerning the conditioning process perse are both premature and incomplete.

Second, Cook (1968) presented evidence indicating that response probability is far from satisfactory as a dependent variable when examining classical conditioning of the Cebus monkey eyelid response. He assessed the contribution of nonassociative factors to conditioning performance and demonstrated that the separation of the associative and nonassociative components of the conditioned response, as indicated by response probability, is at best extremely difficult. The reason for this, Cook reported, is that off-trial response rate increases as a function of stimulus presentations perse; the concurrent increase in on-trial response rate yields an increased probability of a response meeting conditioned response criteria, which is insensitive to commonly employed experimental control procedures. Cook concluded that the associative factors of the conditioning paradigm were masked by nonassociative factors when examined in terms of response probability.

Third, Pennypacker (1964) has discussed the utilization of a magnitude measure of the eyelid response, defined as the integral of the amplitude of the blink with respect to time, as the dependent variable in eyelid-conditioning experiments. High correlations between magnitude and response latency and amplitude and a low correlation between magnitude and response frequency were reported. Pennypacker contended that the magnitude measure is superior to frequency data for the assessment of the effects of the conditioning paradigm upon the eyelid response because it is a continuous measure, highly sensitive to changes in the physiological processes underlying the eyelid response.

\section{SUBJECTS}

Twelve young adult male jungle-born Cebus monkeys (Cebus albifrons) served as Ss.

\section{APPARATUS}

The apparatus and general procedure were similar to those described by Pennypacker, King, Achenbach, \& Roberts (1966). Briefly, Ss were placed in a restraining chair modified by the addition of a bucket seat and headholder designed to immobilize S's head and to negate the necessity of attaching plastic posts to S's head as described in the Pennypacker et al paper. The microtorque potentiometer employed in the transduction of the eyelid response was relocated to a fixed mounting position above S's head. The arm of the potentiometer was mechanically linked to the eyelid by means of a length of $.01-\mathrm{cm}$-thick copper wire hooked through a small triangular piece of plastic tape fastened to the monkey's right eyelid. Movements of the potentiometer arm were transformed into voltage changes that were amplified and ink-written on a moving paper record through one channel of a Grass Model 5 polygraph. A second channel of the polygraph was employed to concurrently write on the same record the transformed eyelid movements following their processing by the Grass integrator system. A third channel recorded the presentation of stimuli to $S$

During data collection, the restraining chair containing $S$ was located in an I.A.C. Model 401-A sound-attenuating chamber, with a $60-\mathrm{W}$ light in a conical reflector, located $30 \mathrm{~cm}$ above and $35 \mathrm{~cm}$ behind S's head, provided for illumination. All experimental contingencies and operations were controlled by automatic electromechanical programming equipment. A Schmitt trigger was operative on all avoidance trials and, when activated by a CR, terminated the $\mathrm{CS}$ and precluded presentation of the aversive stimulus.

Each CS was a tone of $1,500-\mathrm{msec}$ duration at $80 \mathrm{~dB}$ intensity of either $600 \mathrm{~Hz}$ or $1,800 \mathrm{~Hz}$ delivered through either of two $10-\mathrm{cm}$-diam speakers mounted on each side of the restraining chair $14 \mathrm{~cm}$ from S's ear. The reinforcing stimulus was a 2-psi air puff of 200-msec duration delivered to the comer of the right eye. The mean intertrial interval was $30 \mathrm{sec}$, with a range from 10 to $50 \mathrm{sec}$.

\section{DESIGN}

The 12 Ss were assigned randomly to one of three equal-sized groups labeled differential classical (DC), differential avoidance (DA), and differential 
classical-avoidance (DCA). Group DC experienced differential classical conditioning with $\mathrm{CS}+$ always followed by the UCS and CS - never so followed. In Group DA, a conditioned response in the presence of $\mathrm{CS}+$ resulted in the termination of that stimulus and preclusion of presentation of the aversive stimulus. On CS - trials, that stimulus was always of full duration, and the aversive stimulus was never presented, regardless of any response. For Group DCA, CS classical was always followed by the UCS regardless of responding, while responses in the presence of $\mathrm{CS}_{\text {avoidance }}$ terminated that stimulus and prevented the occurrence of the aversive stimulus. The two components of each experimental paradigm were arranged in a restricted random sequence, with each of the components appearing an equal number of times in each block of 50 trials. Within each experimental group, CS frequency and source and reinforcement contingency were counterbalanced. The experiment was performed in four replications; each replication involved one $S$ from each experimental group.

\section{PROCEDURE}

During the course of the experiment, Ss were housed in individual cages with ad lib water and Purina monkey chow. Fresh fruit was provided after each daily session. At the beginning of each session, $S$ was placed in the restraining chair in the experimental chamber, and all recording equipment was attached. Prior to the initiation of the experimental manipulations, each $S$ was subjected to

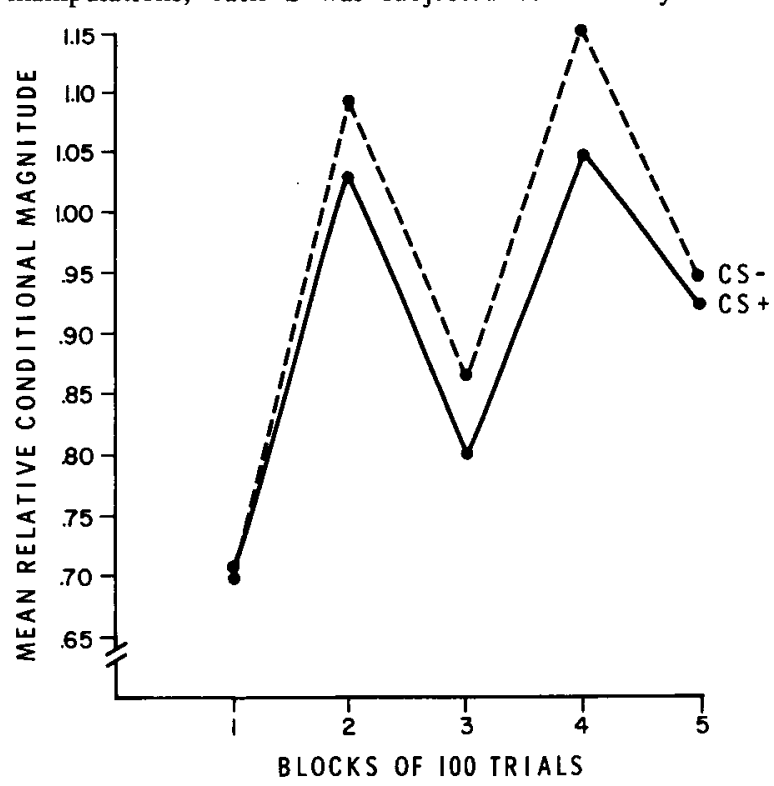

Fig. 1. Differential classical-conditioning performance plotte.t in terms of mean

relative conditional magnitude. three daily chair adaptation sessions of 1 h duration. Commencing on the 4th day and for each of 5 days, $S$ was allowed a $10-\mathrm{min}$ adaptation period and was then presented 100 acquisition trials.

\section{RESULTS}

A conditioned response was defined as any deflection in the direction of closure that exceeded $1 \mathrm{~mm}$ on the ink-written record and was of greater than 150-msec latency. The written record was constantly monitored and the baseline adjusted to counteract the effects of shifts of gaze upon the sensitivity of the Schmitt trigger. Analyses of variance of the response-frequency data failed to reveal either acquisition or differentiation for any of the three groups. However, two of the four $\mathrm{Ss}$ in Group DC exhibited some evidence of differentiation, suggesting that under more optimal circumstances, differentiation within this paradigm might be demonstrable in the Cebus monkey. It should be noted that this is the condition in which Massaro and Moore reported the greatest differentiation.

Of principle interest are the results of the analyses of the CR magnitude data derived from the Grass integrator system. The mean magnitude of five spontaneous blinks during the 10-min adaptation period at the beginning of each session was determined for each $S$ and served as the basis for the conversion of each response to a proportion of this value. These values were termed relative magnitudes and served to eliminate day-to-day and monkey-to-monkey variations due to differences in calibration, potentiometer coupling, and eyelid size. In order to eliminate the contribution of response frequency to the magnitude analyses, trials in which no response occurred were excluded from consideration. The resultant measures were averaged over trial blocks and termed "Mean Relative Conditional Magnitudes" (MRCM). All data were plotted by daily blocks (100 trials), and analyses of variance were performed on each group by 50 trial blocks.

Figure 1 shows the MRCMs for Group DC. Analysis of variance failed to reveal either a significant differentiation $[F(1,3)=5.450, p \leqslant .10]$ or a significant trials effect $[F(9,27)=2.046 . \quad p \leqslant .10]$. However, the probability levels do suggest that under more optimal circumstances or with an enlarged $\mathrm{N}$, both differentiation and acquisition might well be reflected by a magnitude analysis of responding. It should be noted that the tendency is for those responses occurring in the presence of CSt to be of smaller magnitude than those occurring during CS-.

Figure 2 shows the MRCMs for Group DA. Neither differentiation $[F(1,3)<1, p>.10]$ nor trials $[F(9,27)=1.091, \quad p>.10]$ were significant.

Figure 3 shows the MRCMs for Group DCA. Differentiation was significant $[F(1,3)=10.781, \quad p<.01]$, with the responses occurring during the classical trials smaller than those on the avoidance trials. The trials effect failed to reach significance $[F(9,27)=1, p>.10]$.

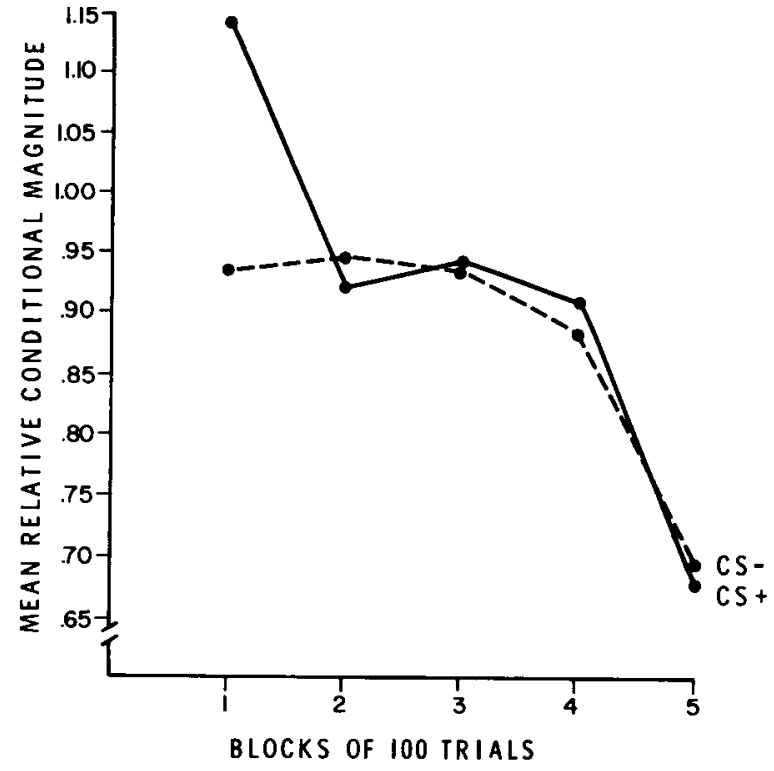

Fig. 2. Differential avoidanceconditioning performance plotted in terms of mean relative conditional magnitude. 
Fig. 3. Differential classical-avoidanceconditioning performance plotted in terms of mean relative conditional magnitude.

The above findings support Pennypacker's (1964) contention that a magnitude analysis of eyelid-conditioning performance is a viable alternative to the commonly employed response-probability measure. The failure of the response-probability measure to index either acquisition or differentiation, most probably the result of the pronounced struggling induced in the Cebus monkey by the restraint procedure employed and concommitant high spontaneous blink rate, and the nonassociative factors discussed by Cook (1968), is partially remedied by the magnitude analysis. Although no group demonstrated clear acquisition evidence when examined in terms of MRCMs, Group DC tended to show an acquisition effect. Moreover, the tendency for Group DC to evidence differentiation and the significant differentiation demonstrated by Group DCA indicate that a complex acquisition process not revealed by the probability data was indeed operative. In addition, the differentiation of Group DCA contradicts Massaro \& Moore's (1967) contention that the two responses cannot develop simultaneously in the same organism.

In each of the above instances, the conclusions drawn concerning the effect of experimental manipulations are determined by the dependent variable utilized. Such a

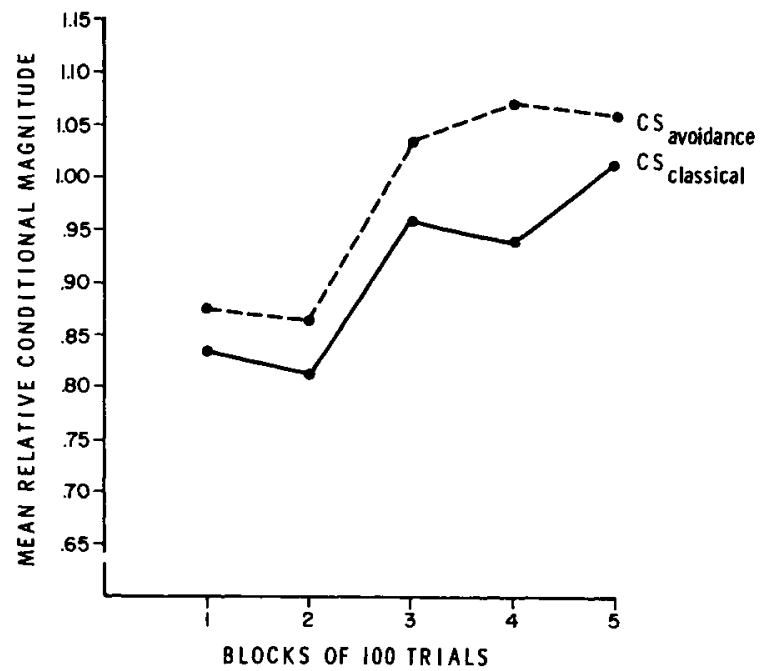

disparity should not discourage the introduction of a little-employed measure in conditioning studies when practical considerations so dictate; to the contrary, such findings indicate that such measures have been too long ignored, and that general statements concerning the conditioning process must await their thorough examination.

\section{REFERENCES}

COOK, W. A. The contribution of nonassociative factors to cebus monkey eyelid conditioning performance. Unpublished doctoral dissertation, University of Florida, 1968.

FISHBEIN, H. F., \& GORMEZANO, I. Effects of differential instructions, differential payoffs, and the presence or absence of feedback on the percentage, latency, and amplitude of the conditioned eyelid response. Joumal of Experimental Psychology, 1962, 63, 487-494.
LEVEY, A. B., \& MARTIN, I. Reflex sensitivity and the conditioned eyelid response. Psychonomic Science, 1967, 8, 153-154.

MASSARO, D. W., \& MOORE, J. W. Differential classical and avoidance eyelid conditioning. Journal of Experimental Psychology, 1967, 75, 151-157.

PENNYPACKER, H. S. Measurement of the conditioned eyelid reflex. Science, 1964, 144, 1248-1249.

PENNYPACKER, H. S., KING, F. A., ACHENBACH, K. E., \& ROBERTS, L. An apparatus and procedure for conditioning the eye-blink reflex in the squirrel monkey. Journal of the Experimental Analysis of Behavior, 1966, 9, 601-604.

\section{NOTE}

1. This research was jointly supported by USPHS Grants MH-08887 and HE-06379, the latter a primate facilities support grant under the direction of A. F. Moreland, D.V.M. 